\title{
Virtual Line Shafting-Based Total-Amount Coordinated Control of Multi-Motor Traction Power
}

\author{
Jing He $\mathbb{D}^{\mathbb{D}},{ }^{1}$ Xueyuan Chen $\mathbb{D}^{1},{ }^{1}$ Songan Mao, ${ }^{2}$ Changfan Zhang $\mathbb{D}^{1},{ }^{1}$ and Jianhua Liu $\mathbb{D}^{1}$ \\ ${ }^{1}$ College of Electrical and Information Engineering, Hunan University of Technology, Zhuzhou 412000, China \\ ${ }^{2}$ School of Electrical and Computer Engineering, Purdue University, 465 Northwestern Ave, West Lafayette, IN 47907, USA \\ Correspondence should be addressed to Jianhua Liu; jhliu@hut.edu.cn
}

Received 25 May 2019; Revised 25 August 2019; Accepted 25 September 2019; Published 29 January 2020

Academic Editor: Paola Pellegrini

Copyright (C) 2020 Jing He et al. This is an open access article distributed under the Creative Commons Attribution License, which permits unrestricted use, distribution, and reproduction in any medium, provided the original work is properly cited.

\begin{abstract}
This paper investigates a virtual line shafting-based total-amount coordinated control method of multi-motor traction power to solve the traffic safety problem caused by train traction power loss. This method considers the total amount instead of the synchronous control amongst single motors in a multi-motor control system. Firstly, a block diagram of the proposed method is built. Secondly, on the basis of this diagram, an accurate system model with parameter perturbations is constructed. Thirdly, a virtual controller is designed to quickly adjust the output torque of the virtual motor and to realise a tracking control of the reference torque. A totalamount coordinated control strategy based on the integral sliding mode is also designed to keep the total traction power of the multi-motor system constant under uncertain and unknown disturbances. Lyapunov stability theory is used to prove the system stability. The simulation and experiment results verify the effectiveness of the virtual controller and the total-amount coordinated control strategy in guaranteeing system robustness under disturbances and parameter perturbations.
\end{abstract}

\section{Introduction}

Train safety is an important issue in transportation systems [1]. One key feature of train control technologies is traction system control [2-5]. Previous studies have transformed the traction system into a multi-agent system and identified consensus problem as the basis and core of maintaining a coordinated control of this system [6,7]. Consensus algorithm has been widely studied by experts through system-local information exchange and a simple controller structure to ensure that each state in a large and complex multi-agent system is progressively constant [8-15]. In recent years, studies on consensus issues have yielded important results. For instance, Bai et al. used graph theory and performed a fractional stability analysis to study the consensus of the fractional multi-agent system (quality) in a reference state [16]. Yang designed a robust controller to solve the distributed consensus tracking control problem of high-order uncertain nonlinear systems on directed graphs [17]. Wu and $\mathrm{Su}$ investigated the positive relationship of edges with multi-input and multi-output positive dynamics for directed and undirected networks [18]. However, these studies have only focused on the consensus amongst individuals within the system. In some engineering situations, individuals do not need to synchronise with one another but must keep the total output traction power constant [19]. For example, when a train wheel set has an idling failure, the traction power of the wheel set will be lost, which in turn leads to a total traction power loss for the train. To ensure a safe operation of the train, its total traction power must stay unchanged. To address this problem, Zhang et al. used the terminal sliding mode control method to transform the total traction power problem into an error system convergence problem [19] and then designed a consensus-based totalamount cooperative tracking control protocol based on the disturbance observer [7].

Electronic virtual line-shafting (ELS) has been widely used in multi-motor synchronic control systems. Lin et al. proposed ELS control based on the nonlinear reaching law observer [20]. Zhang et al. proposed a new ELS control method of equivalent load torque observer based on observer technology [21]. Andert et al. investigated an ELS algorithm for dynamic synchronic motion control in a dynamic test [22]. However, these studies do not consider the feedback mechanism of ELS in the 


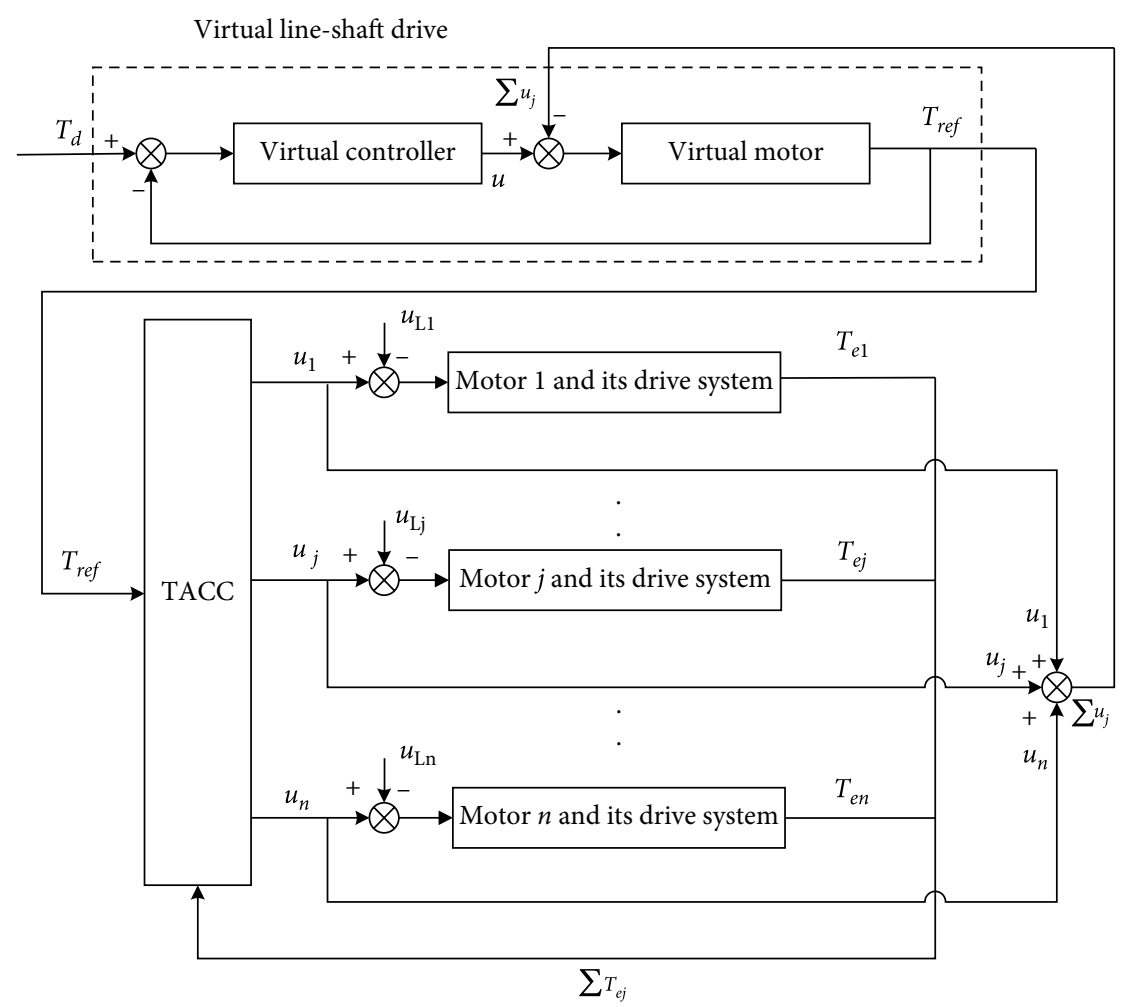

FIGURE 1: Block diagram of the virtual line shafting-based TACC method for controlling multi-motor traction power.

consensus problem to guarantee the dynamic tracking effect of the system.

Following previous research, this study proposes a virtual line shafting-based total-amount coordinated control (TACC) method for controlling multi-motor traction power. The contributions of this work are twofold. Firstly, a block diagram of the proposed method is built and a virtual controller is used to quickly adjust the output torque of the virtual motor and to speed up the system tracking. Secondly, a TACC strategy for traction power is designed based on the proposed integral sliding mode to guarantee that the total traction power is kept constant.

The rest of this paper is organised as follows. Section 2 describes the system and the system target design. Section 3 establishes the multi-traction motor model. Section 4 presents the virtual controller and the TACC strategy. Section 5 presents the simulation results. Section 6 concludes the paper.

\section{System Description}

This paper designs a virtual line shafting-based TACC method for controlling multi-motor traction power. When a motor loses traction power, this method senses the situation through the total feedback of system, and then the controller is used to maintain the system total traction power. Figure 1 presents a block diagram of the proposed method.

As shown in Figure 1, closed-loop system comprises a given torque $T_{d}$, virtual line-shafting, TACC strategy, motors and their drive systems. $T_{r e f}$ is the output torque of the virtual motor, $u$ is the output of the virtual controller, $u_{j}$ is the output of TACC and $u_{L j}$ is the external disturbance in the multi-motor system $(j=1,2, \ldots, n)$. When losing traction power, the voltage of a motor can change. The feedback loop feedbacks the total voltage of all motors to the virtual line-shafting, which in turn adjusts the total traction torque of the motor by adjusting the sum of the voltages, thereby ensuring that the total traction torque of all motors dynamically tracks the given torque of the system and keeps the total-amount of traction power constant. The goal of this paper can be summarised as follows. After a motor loses traction power, the multi-motor system can keep the total-amount of traction power constant. In other words, after a motor is affected by a disturbance, the controller can keep the output of total rotation constant.

\section{System Model}

Given a multi-motor system that comprises multiple permanent magnet traction motors, where motor 0 is a virtual motor and motors $1 \cdots n$ are the follower motors, the following mathematical model of the $j$ th motor is established [7]:

$$
\begin{aligned}
& L_{j} \frac{d i_{j}}{d t}=-R_{j} i_{j}-k_{e j} k_{t j} \omega_{j}+u_{j} \\
& \left(J_{1 j}+k_{t j}^{2} J_{0 j}\right) \dot{\omega}_{j}=-\left(b_{1 j}+k_{t j}^{2} b_{0 j}\right) \omega_{j}+T_{e j}-T_{L j} \\
& T_{e j}=k_{t j} k_{m j} i_{j}, \\
& (j=1,2, \ldots, n),
\end{aligned}
$$

Each symbol in the above formula is defined in Table 1. 
TABLE 1: Definition of each symbol in the mathematical model.

\begin{tabular}{lc}
\hline Symbols & Definition \\
\hline$R_{j}$ & Resistance of armature circuit \\
$L_{j}$ & Inductance of armature circuit \\
$i_{j}$ & Motor current \\
$\omega_{j}$ & Output angular velocity of gearbox of $j$ th motor \\
$u_{j}$ & The voltage applied to the input terminals of the \\
$k_{e j}$ & armature circuit \\
$k_{t j}$ & Back electromotive force constant \\
$J_{0 j}$ & Speed reduction ratio of the gearhead \\
$J_{1 j}$ & Inertia moments of the motor \\
$b_{0 j}$ & Inertia moments of the gearhead \\
$b_{1 j}$ & Viscous damping coefficients of the motor \\
$k_{m j}$ & Viscous damping coefficients of the gearhead \\
$T_{L j}$ & Motor torque constant \\
$T_{e j}$ & The bounded load torque \\
\hline
\end{tabular}

Given that the output angular velocity is differentially flat, all variables and control inputs can be expressed by their output angular velocity [23]. Taking the angular acceleration as the state variable, i.e. $x_{1 j}=\omega_{j}, x_{2 j}=\dot{x}_{1 j}, x_{3 j}=T_{e j}$, the equation in the ideal system state is $[7,24]$

$$
\begin{aligned}
& \dot{x}_{1 j}=x_{2 j} \\
& \dot{x}_{2 j}=-a_{0 j} x_{1 j}-a_{1 j} x_{2 j}+b_{j} u_{j}+f_{j} \\
& \dot{x}_{3 j}=-J_{e q j} a_{0 j} x_{1 j}+\left(b_{e q j}-J_{e q j} a_{1 j}\right) x_{2 j}+J_{e q j} b_{j} u_{j}+J_{e q j} f_{j}+\dot{T}_{L j} \\
& (j=1,2, \ldots, n),
\end{aligned}
$$

where $J_{e q j}=J_{1 j}+k_{t j}^{2} J_{0 j}, \quad b_{e q j}=b_{1 j}+k_{t j}^{2} b_{0 j}, a_{0 j}=\left(k_{t j}^{2} k_{m j} k_{e j}+\right.$ $\left.R_{j} b_{e q j}\right) / J_{e q j} L_{j}, \quad a_{1 j}=b_{e q j} / J_{e q j}+R_{j} / L_{j}, \quad b_{j}=k_{t j} k_{m j} / J_{e q j} L_{j}$, $f_{j}=\left(-R_{j} / J_{e q j} L_{j}\right) T_{L_{j}}-\left(1 / J_{e q j}\right) \dot{T}_{L_{j}}$.

Given that the parameters of the motor during the actual operation, including starting and braking, are time-varying [7], $a_{0 j}=\bar{a}_{0 j}+\Delta a_{0 j}, a_{1 j}=\bar{a}_{1 j}+\Delta a_{1 \underline{j}}, b_{e q j}=\bar{b}_{e q j}+\Delta b_{e q j}$ and $b_{j}=\bar{b}_{j}+\Delta b_{j}$, where $\bar{a}_{0 j}, \bar{a}_{1 j}, \bar{b}_{e q j}$ and $\bar{b}_{j}$ represent the nominal values of $a_{0 j}, a_{1 j}, b_{e q j}$ and $b_{j}$, respectively, and $\Delta a_{0 j}, \Delta a_{1 j}, \Delta b_{e q j}$ and $\Delta b_{j}$ represent the uncertain perturbation values. Therefore, the closeness to the actual system model of the project can be formulated as

$$
\begin{aligned}
& \dot{x}_{1 j}=x_{2 j} \\
& \dot{x}_{2 j}=-\bar{a}_{0 j} x_{1 j}-\bar{a}_{1 j} x_{2 j}+\bar{b}_{j} u_{j}+d_{1 j} \\
& \dot{x}_{3 j}=-J_{e q j} \bar{a}_{0 j} x_{1 j}+\left(\bar{b}_{e q j}-J_{e q j} \bar{a}_{1 j}\right) x_{2 j}+J_{e q j} \bar{b}_{j} u_{j}+d_{2 j} \\
& (j=1,2, \ldots, n),
\end{aligned}
$$

where $d_{1 j}=-\Delta a_{0 j} x_{1 j}-\Delta a_{1 j} x_{2 j}+\Delta b_{j} u_{j}+f_{j}, d_{2 j}=-J_{e q j} \Delta a_{0 j} x_{1 j}+$ $\left(\Delta b_{e q j}-J_{e q j} \Delta a_{1 j}\right) x_{2 j}+J_{e q j} \Delta b_{j} u_{j}+J_{e q j} f_{j}+\dot{T}_{L j}$. The parameter perturbation values, $\Delta a_{0 j}, \Delta a_{1 j}, \Delta b_{e q j}, \Delta b_{j}$ and state variables are bounded in engineering practice.

To simplify this model, Equation 3 should be

$$
\dot{\boldsymbol{x}}_{j}=\overline{\boldsymbol{A}}_{j} \boldsymbol{x}_{j}+\overline{\boldsymbol{B}}_{j} u_{j}+\boldsymbol{d}_{j}
$$

where

$$
\boldsymbol{x}_{j}=\left[x_{1 j}, x_{2 j}, x_{3 j}\right]^{\mathrm{T}}
$$

$$
\begin{gathered}
\overline{\boldsymbol{A}}_{j}=\left(\begin{array}{ccc}
0 & 1 & 0 \\
-\bar{a}_{0 j} & -\bar{a}_{1 j} & 0 \\
-J_{e q j} \bar{a}_{0 j} & \bar{b}_{e q j}-J_{e q j} \bar{a}_{1 j} & 0
\end{array}\right), \\
\overline{\boldsymbol{B}}_{j}=\left[\begin{array}{ll}
0 \bar{b}_{j} & J_{e q j} \bar{b}_{j}
\end{array}\right]^{\mathrm{T}} \\
\boldsymbol{d}_{j}=\left[\begin{array}{lll}
0 & d_{1 j} & d_{2 j}
\end{array}\right]^{\mathrm{T}}
\end{gathered}
$$

is the time-varying compound disturbance.

The control objectives of this paper for multi-motor systems can be expressed as

$$
\lim _{t \rightarrow \infty}\left(\sum_{j=1}^{n} x_{3 j}-T_{d}\right)=0,
$$

where $t$ denotes time.

\section{Controller Design}

The proposed method can be divided into two parts, namely, the tracking control of virtual line-shafting and the TACC of follower motors. In the first part, the control target is to design a tracking control algorithm for the virtual motor in order for the error between the output torque of the virtual motor and the given torque of the system to converge to zero, that is, $\lim _{t \rightarrow \infty}\left\|T_{d}-T_{r e f}\right\|=0$. In the second part, the control target is to design a tracking control algorithm for the multi-motor system to guarantee that the error between the output torque of the virtual motor and the total traction torque of the multi-motor system converges to zero, that is, $\lim _{t \rightarrow \infty}\left\|T_{r e f}-\sum_{j=1}^{n} T_{e j}\right\|=0$.

4.1. Virtual Controller Design. In the ELS control strategy, the virtual electronic-shaft functions the same as the physical line shaft in the traditional mechanical shaft and can drive the other slave shafts by transmitting the control signal [21, 23]. The control strategy of virtual line-shafting lies in its feedback mechanism [20]. In this paper, the feedback characteristics of the control strategy of virtual line-shafting are used. When the motor is disturbed, the virtual line-shafting perceives the total voltage value through the total feedback of the system and then adjusts the total output torque until reaching a steady state whilst ensuring that the output value dynamically tracks the reference value given by the system.

As shown in Figure 1, virtual line shafting consists of a virtual controller and a virtual motor. The virtual motor is virtually set, and its parameters are known and invariable. The PI controller $u$ is then designed to achieve the control target, where $u=k_{P}\left(T_{d}-T_{r e f}\right)+k_{I} \int\left(T_{d}-T_{r e f}\right) d t k_{P}$ and $k_{I}$ are the positive constants to be designed [25].

4.2. TACC Design. The multi-motor traction power system requires a high-precision TACC and the highly complicated model of the actual motor. A sliding mode controller is designed. Given its simplicity, high real-time performance and 
strong robustness, this controller has been widely used in highprecision tracking control $[21,26,27]$. To guarantee that the total traction torque of the multi-motor system dynamically tracks the output torque of the virtual motor, the error between these two is denoted by $e$. The total-amount coordinated tracking error of the traction power of the system can be formulated as

$$
\begin{aligned}
e & =\sum_{j=1}^{n} T_{e j}-T_{r e f} \\
& =T_{e 1}+\cdots+T_{e j}+\cdots+T_{e n}-T_{r e f} \\
& =x_{31}+\cdots+x_{3 j}+\cdots+x_{3 n}-T_{r e f} .
\end{aligned}
$$

Take the derivative of Equation (6)

$$
\begin{aligned}
\dot{e}= & \dot{T}_{e 1}+\cdots+\dot{T}_{e j}+\cdots+\dot{T}_{e n}-\dot{T}_{r e f} \\
= & -J_{e q 1} \bar{a}_{01} x_{11}+\left(\bar{b}_{e q 1}-J_{e q 1} \bar{a}_{11}\right) x_{21}+J_{e q 1} \bar{b}_{1} u_{1}+d_{21} \\
& +\cdots \\
& +\left(-J_{e q j} \bar{a}_{0 j} x_{1 j}+\left(\bar{b}_{e q j}-J_{e q j} \bar{a}_{1 j}\right) x_{2 j}+J_{e q j} \bar{b}_{j} u_{j}+d_{2 j}\right) \\
& +\cdots \\
& +\left(-J_{e q n} \bar{a}_{0 n} x_{1 n}+\left(\bar{b}_{e q n}-J_{e q n} \bar{a}_{1 n}\right) x_{2 n}+J_{e q n} \bar{b}_{n} u_{n}+d_{2 n}\right)-\dot{T}_{r e f} .
\end{aligned}
$$

Select the integral sliding surface

$$
s=c \int_{0}^{t} e(t) d t+e(t),
$$

where $c$ is a positive constant.

Design the TACC protocol as

$$
\begin{aligned}
u_{j}= & -J_{e q j}^{-1} \bar{b}_{j}^{-1}\left[-J_{e q j} \bar{a}_{0 j} x_{1 j}+\left(\bar{b}_{e q j}-J_{e q j} \bar{a}_{1 j}\right) x_{2 j}+c x_{3 j}\right. \\
& \left.-\frac{1}{n} \dot{T}_{r e f}-\frac{c}{n} T_{r e f}+\left(D_{j}+\varepsilon_{j}\right) \operatorname{sgn}(s)\right], \\
& (j=1,2, \ldots, n),
\end{aligned}
$$

where $\varepsilon_{j}(j=1,2, \ldots, n)$ is a positive constant, $\operatorname{sgn}(s)$ is a sign function, $D_{j}$ is a known non-negative value and $D_{j} \geq\left|d_{2 j}\right|$.

Theorem 1. Consider the multi-motor system described in Equation (4). If the integral sliding mode controller of Equation (9) is devised, then this controller can guarantee that the total traction output torque of the multi-motor system dynamically tracks the virtual motor output torque, that is, errorecan asymptotically converge to zero.

Proof of Theorem 1. Select the Lyapunov function

$$
V=\frac{1}{2} s^{2}
$$

Take the derivative of Equation (10)

$$
\begin{aligned}
\dot{V}= & s \dot{s} \\
= & s\left[c\left(x_{31}+\cdots+x_{3 j}+\cdots+x_{3 n}-T_{r e f}\right)\right. \\
& +\left(-J_{e q 1} \bar{a}_{01} x_{11}+\left(\bar{b}_{e q 1}-J_{e q 1} \bar{a}_{11}\right) x_{21}+J_{e q 1} \bar{b}_{1} u_{1}+d_{21}\right) \\
& +\cdots \\
& +\left(-J_{e q j} \bar{a}_{0 j} x_{1 j}+\left(\bar{b}_{e q j}-J_{e q j} \bar{a}_{1 j}\right) x_{2 j}+J_{e q j} \bar{b}_{j} u_{j}+d_{2 j}\right) \\
& +\cdots \\
& \left.+\left(-J_{e q n} \bar{a}_{0 n} x_{1 n}+\left(\bar{b}_{e q n}-J_{e q n} \bar{a}_{1 n}\right) x_{2 n}+J_{e q n} \bar{b}_{n} u_{n}+d_{2 n}\right)-\dot{T}_{r e f}\right]
\end{aligned}
$$

TABLE 2: Parameters of motors [7].

\begin{tabular}{lcccc}
\hline Parameters & Motor 1 & Motor 2 & Motor 3 & Motor 4 \\
\hline$R(\Omega)$ & 2.4 & 2.5 & 2.3 & 2.2 \\
$L(H)$ & 0.58 & 0.612 & 0.6 & 0.55 \\
$b_{e q}(\mathrm{~N} \cdot \mathrm{m} \cdot \mathrm{s})$ & 0.075 & 0.08 & 0.07 & 0.06 \\
$J_{e q}\left(\mathrm{~kg} \cdot \mathrm{m}^{2}\right)$ & 2.15 & 2.4 & 2.35 & 2.3 \\
$k_{m}(\mathrm{mN} \cdot \mathrm{m} / \mathrm{A})$ & 82 & 82.2 & 81.8 & 81.5 \\
$k_{e}(\mathrm{mv} / \mathrm{rad} / \mathrm{s})$ & 82.31 & 82.322 & 82.3 & 82.2 \\
$k_{t}$ & 7.95 & 8 & 7.9 & 7.89 \\
\hline
\end{tabular}

Substituting Equation (9) into Equation (11) yields

$$
\begin{aligned}
\dot{V}= & s\left[d_{21}+\cdots+d_{2 j}+\cdots+d_{2 n}\right. \\
& -\left(D_{1}+\varepsilon_{1}\right) \operatorname{sgn}(s) \\
& \left.-\cdots-\left(D_{j}+\varepsilon_{j}\right) \operatorname{sgn}(s)-\cdots-\left(D_{n}+\varepsilon_{n}\right) \operatorname{sgn}(s)\right] \\
\leq & s\left[-\varepsilon_{1} \operatorname{sgn}(s)-\cdots-\varepsilon_{j} \operatorname{sgn}(s)-\cdots-\varepsilon_{n} \operatorname{sgn}(s)\right] \\
= & -\left(\varepsilon_{1}+\cdots+\varepsilon_{j}+\cdots+\varepsilon_{n}\right)|s| \\
\leq & 0 .
\end{aligned}
$$

Both the sliding mode arrival condition and Lyapunov stability theory indicate that the error $e$ can asymptotically converge to zero $[28,29]$. The $s /(\delta+|s|)$ function is typically used instead of the switching function $\operatorname{sgn}(s)$, which can effectively suppress the chattering aroused by the sliding mode variable structure switching action to the control system $[23,30]$. Amongst them, $\delta$ is a positive constant with a small value.

\section{Simulation and Experiment}

To verify the proposed control algorithm, a mathematical simulation and a semi-physical simulation experiment are performed. Table 2 shows the motor parameters used in the simulation and experiment and the parameter settings of resistance $R$, inductance $L$, equivalent viscous friction coefficient $b_{e q}$, equivalent motor rotation inertia $J_{e q}$, motor torque coefficient $k_{m}$, back electromotive force constant $k_{e}$ and gearbox ratio $k_{t}$. Motor 1 , which has the smallest rotation inertia, is chosen as the virtual motor. The corresponding control system parameters are $c=700, \varepsilon_{j}=760, \delta=0.01$, $D_{j}=10$.

5.1. Simulation. The following system time-varying reference command signal is designed [7] to verify the control performance of the multi-motor system under acceleration, uniform speed and deceleration:

$$
T_{d}= \begin{cases}\frac{10}{3} t & t<0.3 \mathrm{~s} \\ 1 & 0.3 \mathrm{~s} \leq t \leq 0.7 \mathrm{~s} \\ -\frac{10}{3}(t-0.7)+1 & 0.7 \mathrm{~s}<t \leq 1 \mathrm{~s} .\end{cases}
$$

The multi-motor system starts and enters an accelerated state at $t<0.3 \mathrm{~s}$, maintains constant speed at $0.3 \mathrm{~s} \leq t \leq 0.7 \mathrm{~s}$ and enters a deceleration state at $0.7 \mathrm{~s}<t \leq 1 \mathrm{~s}$. The motor is assumed to start with parameter perturbation, that is, the system compound interference $d_{2 j} \neq 0$. A pulse interference signal (Figure 2) is applied to motor 2 at $0.2 \mathrm{~s}$, and a high-frequency 


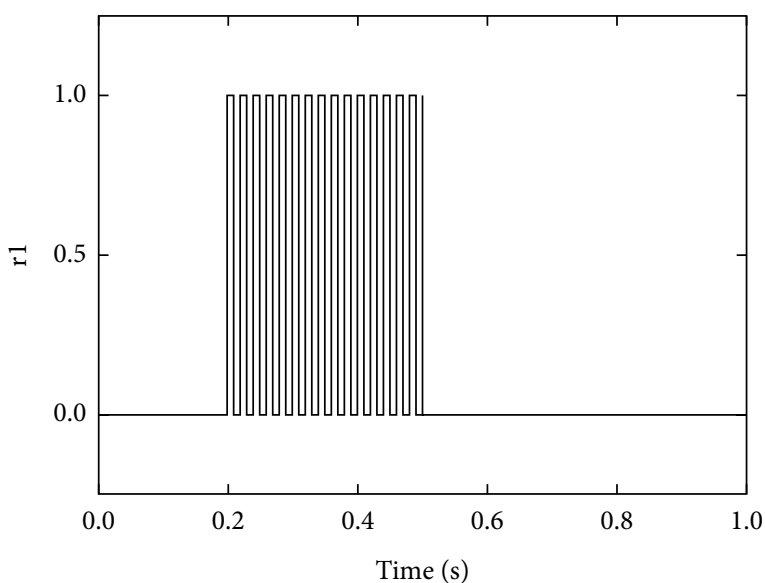

FIgURE 2: Pulse interference signal.

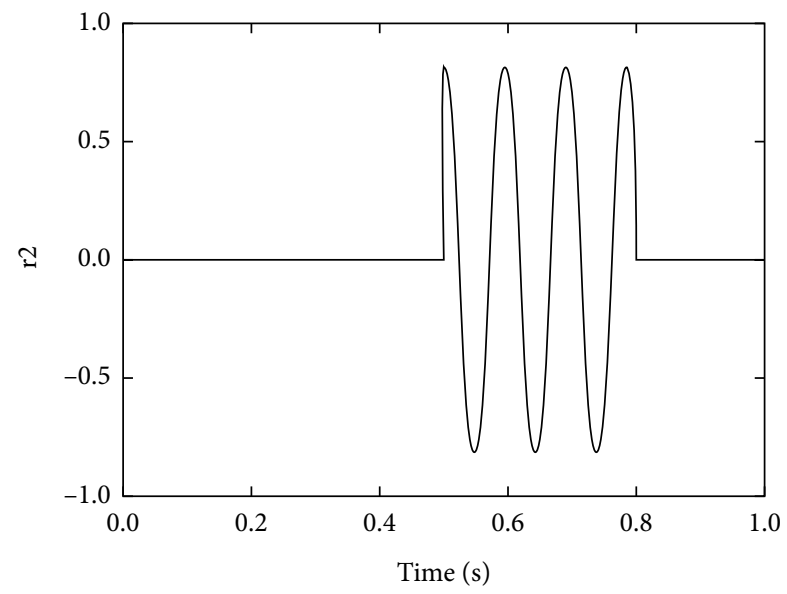

FIGURE 3: High-frequency interference signal.

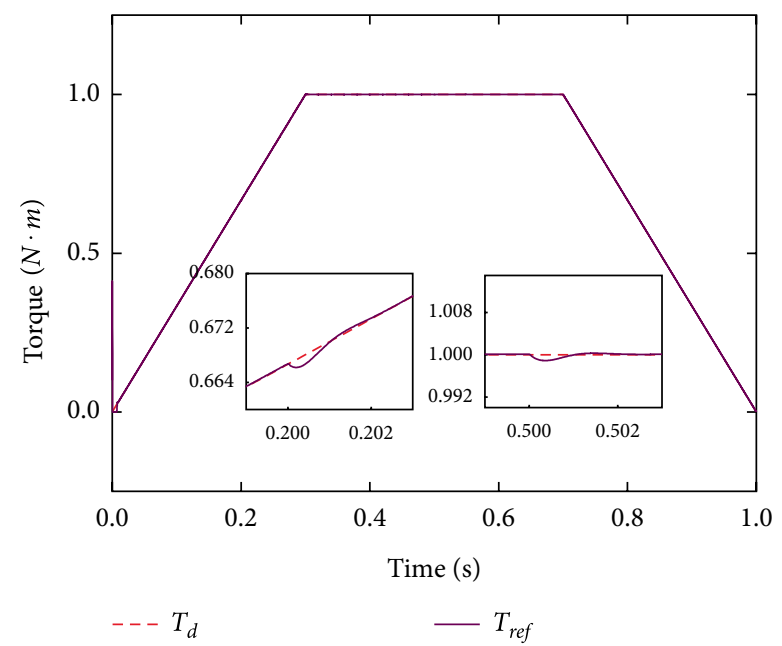

FIGURE 4: Virtual controller tracking effect.

interference signal (Figure 3 ) is applied to motor 3 at $0.5 \mathrm{~s}$. After the motor is disturbed, the control effect of the system occurs, as shown in Figures 4-10. Figures 4 and 5 show the

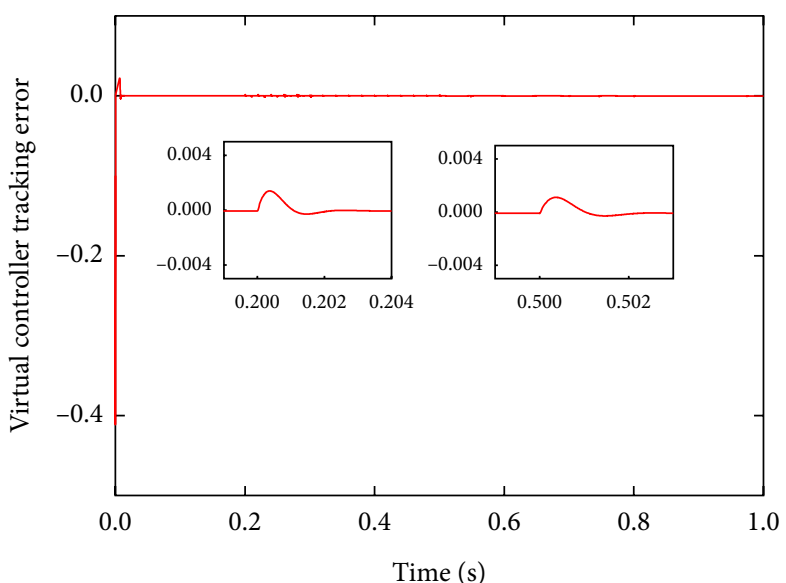

FIGURE 5: Virtual controller tracking error.

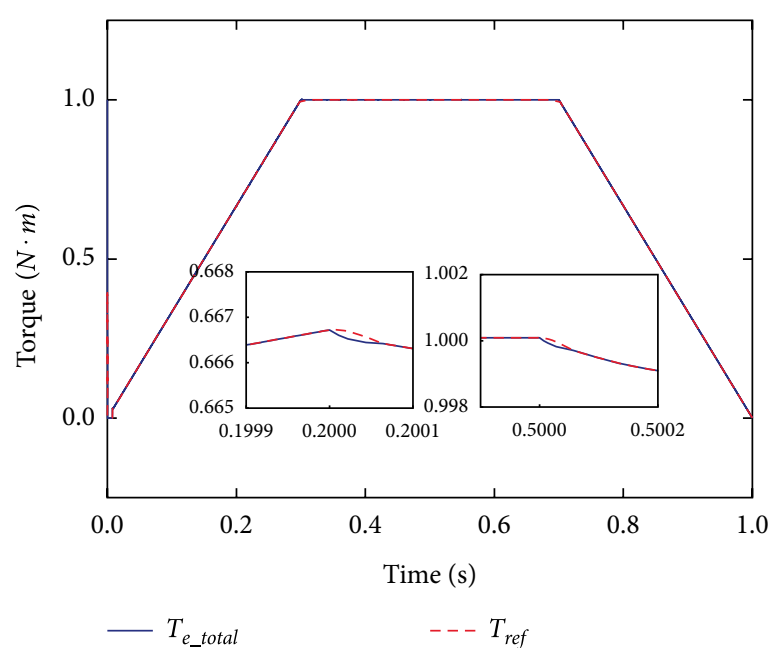

FIGURE 6: TACC tracking effect.

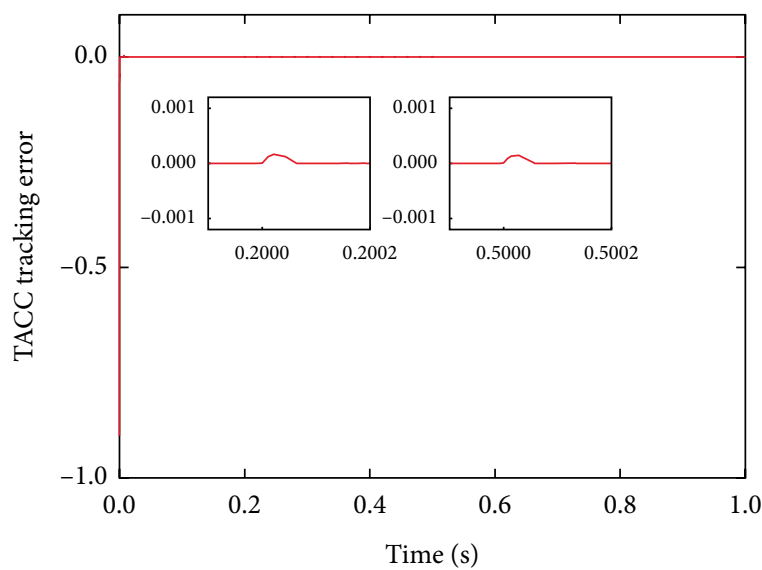

FIGURE 7: TACC tracking error.

tracking effect of the virtual controller and its tracking error. Figures 6 and 7 show the tracking effect of the TACC and its tracking error. Figure 8 shows the output torque and the traction torques of each motor. Figure 9 shows the total-amount 


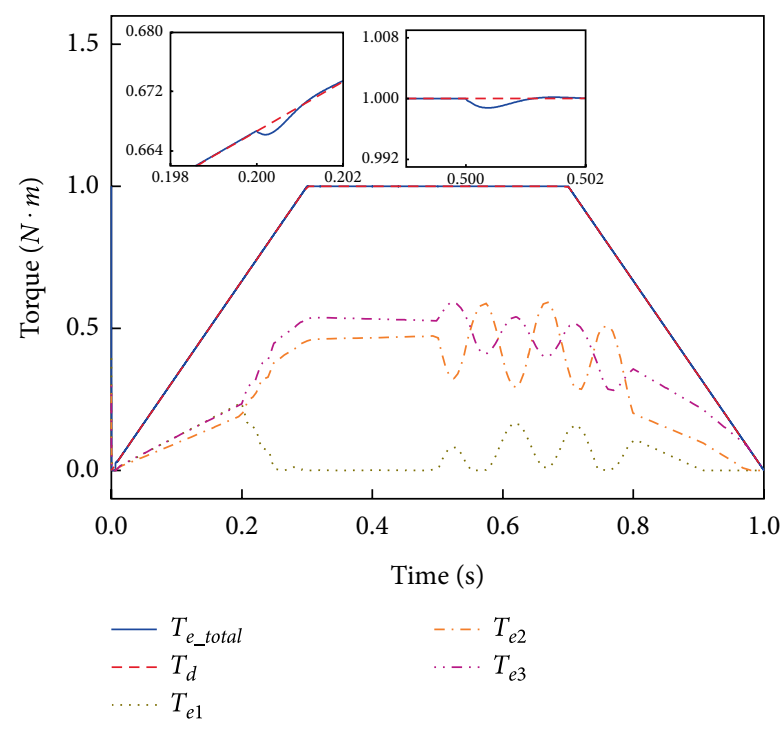

FIGURE 8: Output torque and traction torques of each motor.

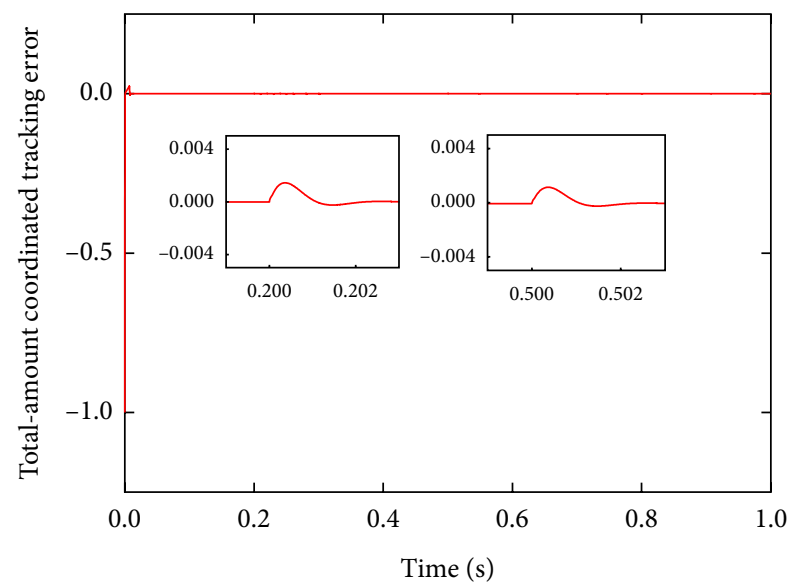

FIGURE 9: Error between the total traction torque and given torque.

coordinated tracking error. Figure 10 shows the given torque of the system, the output torque of virtual line-shafting and the output torque of each motor.

Figures 4 and 5 show the given reference torques (denoted by the dashed line) and the output torque of the virtual motor (denoted by the solid line). The tracking error between these two torques is observed at $0.2 \mathrm{~s}$ and $0.5 \mathrm{~s}$. When the system is disturbed uncertainly, the error value does not exceed $0.2 \%$ and the virtual controller adjustment time is less than $0.002 \mathrm{~s}$. In Figures 6 and 7, the output torque of the virtual motor is denoted by the dashed line, whereas the total output torque is denoted by the solid line. When the system is disturbed, the system tracking error value does not exceed $0.02 \%$, and the TACC adjustment time is less than $0.0002 \mathrm{~s}$.

In Figure 8, the output torques of motors 1 to 3 are denoted by the dotted line, the dotted-dashed line and the double-dotted-dashed line, respectively. When motor 1 is disturbed by a pulse signal at $0.2 \mathrm{~s}$, its output torque decreases. To guarantee a constant total traction power, the output torques of motors
2 and 3 increase. When motor 2 is disturbed by the high-frequency signal at $0.5 \mathrm{~s}$, the output torque of motor 2 decreases, and the output torques of motors 1 and 3 increase. Restricted by the braking command and influenced by the disturbance signal, the output torques of motors 1 to 3 demonstrate a downward trend after $0.8 \mathrm{~s}$. After $0.97 \mathrm{~s}$, the output torques of motors 1 and 2 are equal to 0 , and motor 3 adjusts the output torque to achieve a given torque value and to maintain the consensus of the total traction power. Figure 9 shows that under different signal interferences, the tracking error value does not exceed $0.2 \%$, and the tracking time does not exceed $0.002 \mathrm{~s}$.

The given torque of the system, the output torque of the virtual line-shaft and the output torques of the three motors are plotted on a 3D bar chart in Figure 10(a) and a 3D colour surface map in Figure 10(b). In Figure 10(a), the sum of the cylindrical areas with the same colour in $T_{e j}(j=1,2,3)$ is equal to the corresponding area in $T_{d}$ and $T_{r e f}$. Meanwhile, in Figure 10(b), different colours correspond to different values. The sum of the output torques of the three motors is equal to the virtual line shaft output torque and the given torque of the system. Simulation results indicate that with parameter perturbation, the system still maintains good robustness and convergence performance.

To simulate actual working conditions, a sudden load scenario where a locomotive runs stably is designed. In this simulation, the locomotive is set to run steadily in the constant speed section shown in Figure 8. When $t=0.5 \mathrm{~s}$, the original load increases by $50 \%$ and the duration is $0.2 \mathrm{~s}$. Figure 11 presents the waveform diagram of the given torque and the total output torque of the motors when the proposed virtual line shafting-based TACC method is applied. Figure 12 presents the error value between these two torques. When sudden load is applied, the total output torque of the motors decreases and rapidly increases to the value of the given torque, with the error value not exceeding $2 \%$, and the adjusting time of the controller not exceeding $0.004 \mathrm{~s}$. In sum, the proposed virtual line shafting-based TACC method ensures the strong anti-load interference ability of the system.

5.2. Experiment. To ensure that the system simulation reflects the actual engineering environment as close as possible, a semi-physical simulation experiment is performed.

The apparatus used in this experiment is an RT-lab experimental platform comprising a DSP controller, an OP56000 simulation motor, related connection lines and an upper computer monitoring interface. During the experiment, the system model under the Simulink environment is imported into the RT-lab experimental platform and run in OP56000. Afterwards, the TACC model is imported into the DSP controller, and the results are obtained. The experiment selects the same parameters and conditions described in Section 5.1. Figure 13 shows the RT-Lab experimental platform.

Figure 14 presents the tracking effect of the virtual controller. As shown in this figure, the designed virtual controller can make the virtual line-shafting output torque $T_{\text {ref }}$ accurately track the given torque of the system $T_{d}$. Figure 15 shows the effect diagram of TACC. As shown in this figure, the designed TACC can make the total output torque of the multi-motor 


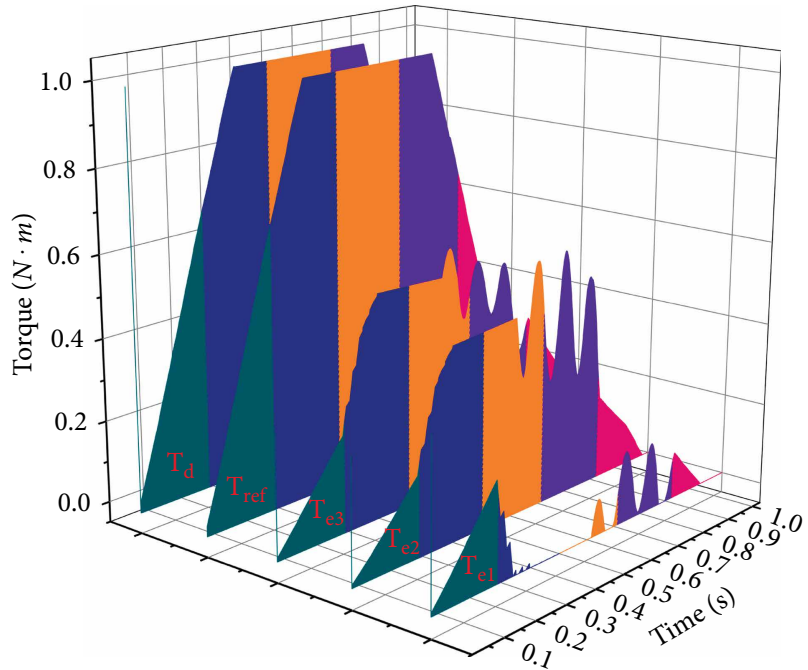

(a)

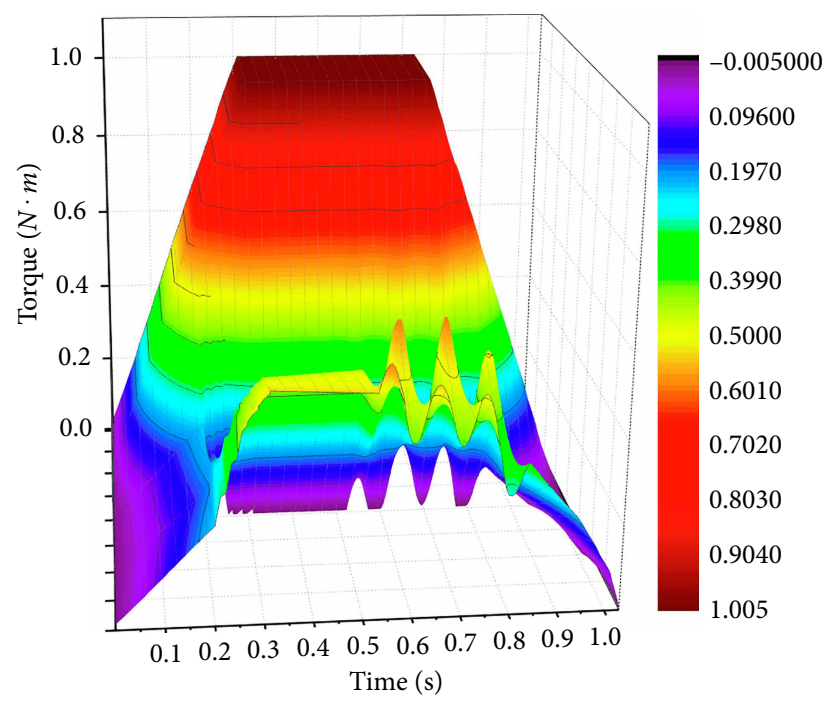

(b)

FIGURE 10: Given torque of the system, output torque of virtual line-shafting and output torque of each motor. (a) 3D bar chart; and (b) 3D colour surface map.

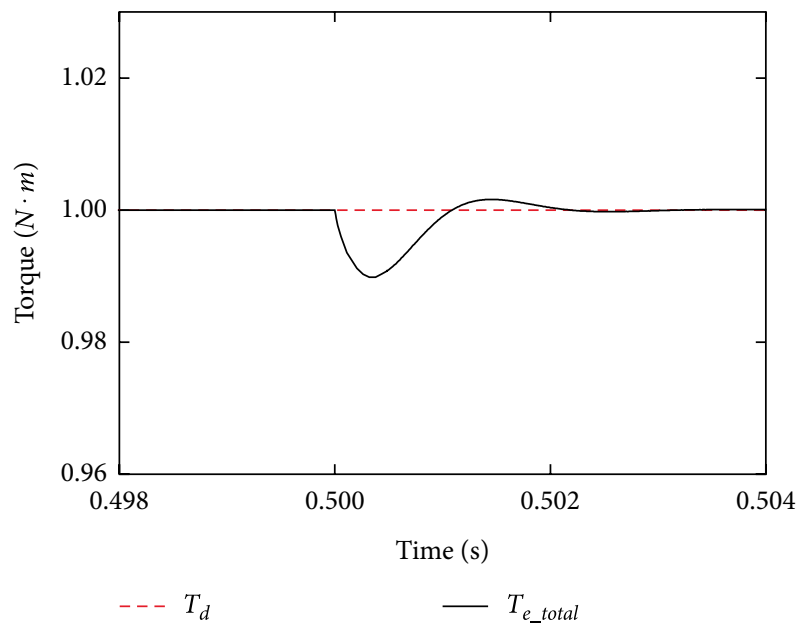

FigURE 11: Given torque and total output torque under sudden load.

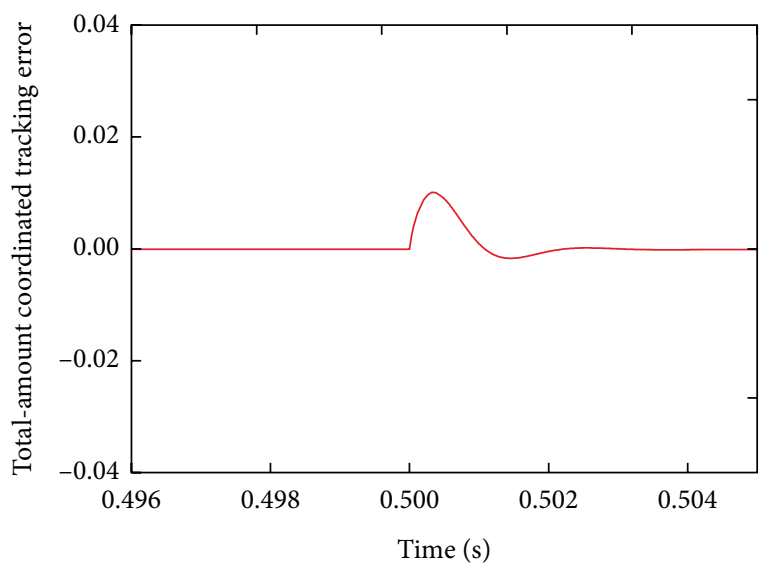

FIGURE 12: Error between the given torque and total output torque under sudden load.

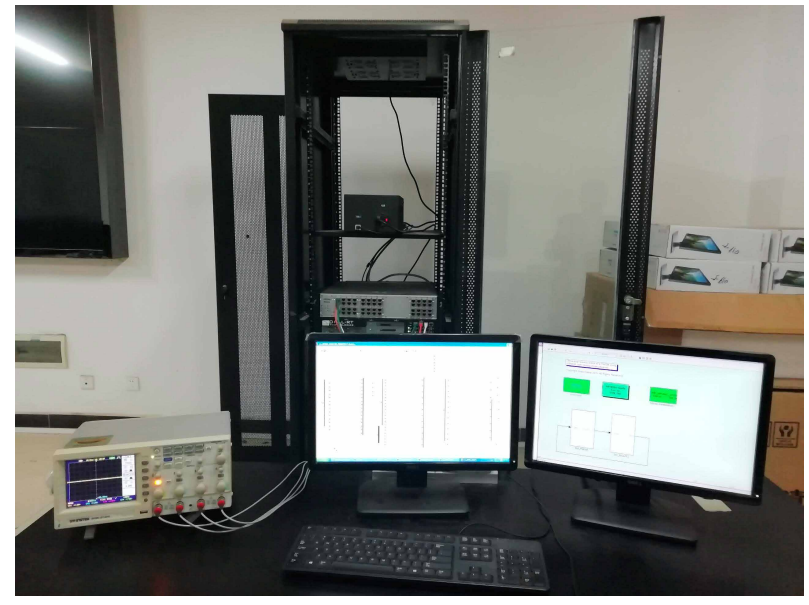

FIGURE 13: RT-Lab experimental platform.

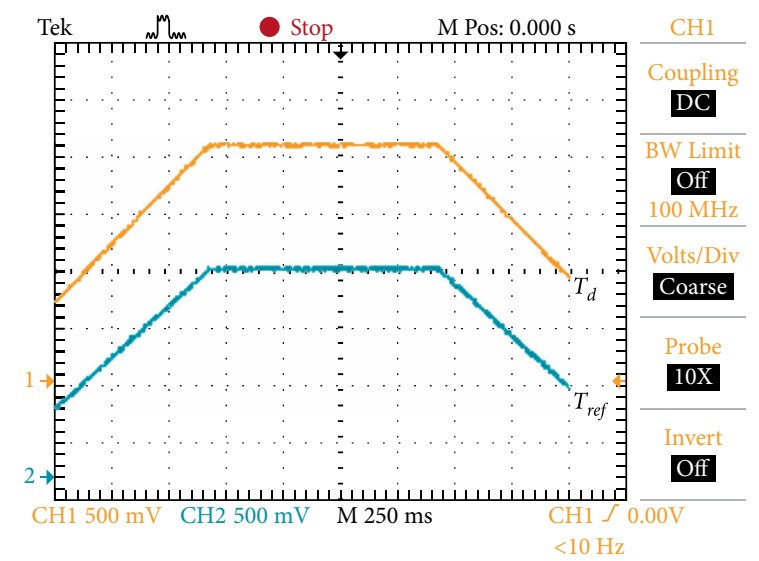

FIgURE 14: Virtual controller tracking effect. 


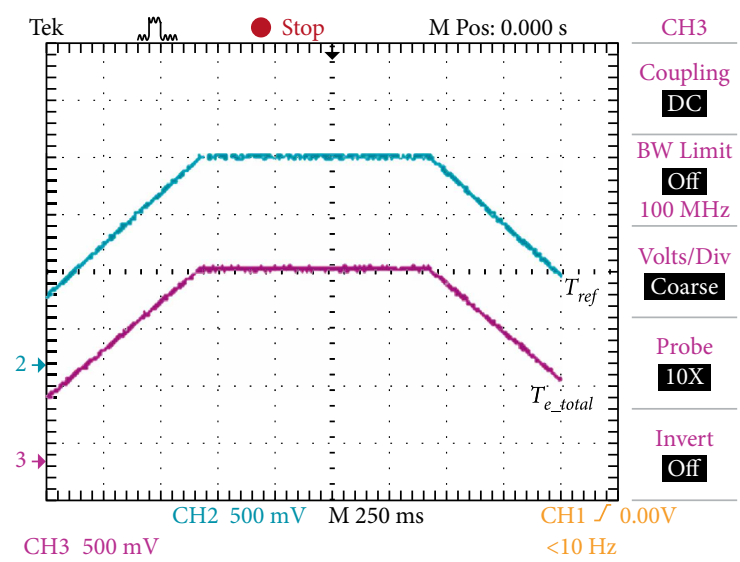

FIGURE 15: TACC tracking effect.

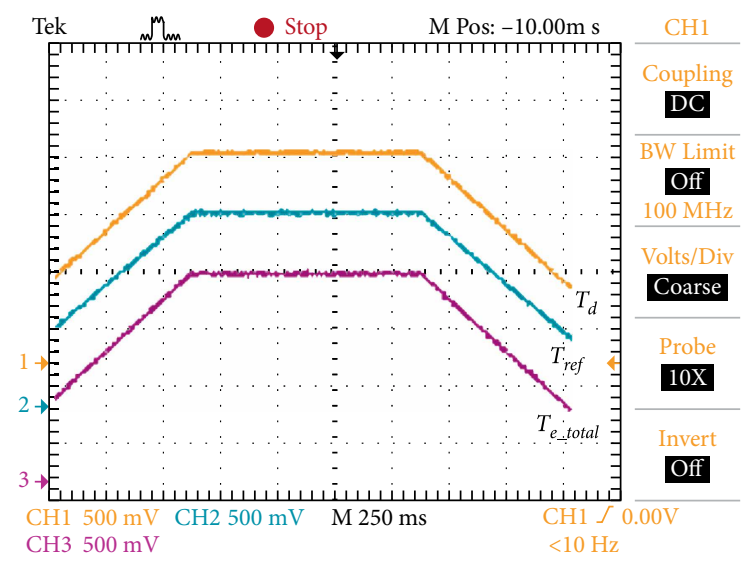

FIGURE 16: Tracking effect of the system.

(i.e. $T_{e}-$ total) consistent with that of virtual line-shafting $T_{r e f}$. Figure 16 presents the tracking effect of the system. Under the action of the two controllers, the total output torque of the multi-motor $T_{e}-$ total can be accurately tracked to the given torque of the system $T_{d}$. Figures 14-16 show that the experimental results are consistent with the simulation results in MATLAB.

\section{Conclusions}

The proposed TACC method can solve the safety problem caused by wheel set idling failure, which leads to the traction power loss of the wheel set. This method allows the system to maintain a constant total traction power in cases of a single traction power loss. Two key findings are obtained. Firstly, the designed virtual controller ensures that the output torque of the virtual motor dynamically tracks the reference torque of the system, thereby shortening the time required for the dynamic process. Secondly, TACC improves the traction performance and ensures that the system shows improved robustness and cooperative tracking performance even when under disturbance. The simulation results prove the effectiveness of the proposed control strategy.

\section{Data Availability}

The data used to support the findings of this study are available from the corresponding author upon request.

\section{Conflicts of Interest}

The authors declare that there is no conflicts of interest regarding the publication of this paper.

\section{Acknowledgments}

This study is supported by the Natural Science Foundation of China (nos. U1934219, 61773159, 61473117), Project of Hunan Provincial Department of Education (no. 19A137), and Hunan Provincial Natural Science Foundation of China (nos. 2017JJ4031, 2018JJ4066).

\section{References}

[1] C. Dong, C. Shao, H. Huang, X. Chen, and N. N. Sze, "Advances in traffic safety methodologies and technologies," Journal of Advanced Transportation, vol. 2018, Article ID 4129582, 2 pages, 2018.

[2] J. Yin, Y. Xie, T. Peng, C. Yang, and Z. Chen, "Current characteristics analysis and fault injection of an early weak fault in broken rotor bar of traction motor," Mathematical Problems in Engineering, vol. 2018, Article ID 4934720, 8 pages, 2018.

[3] K. Keskin and A. Karamancioglu, "Energy-efficient train operation using nature-inspired algorithms," Journal of Advanced Transportation, vol. 2017, Article ID 6173795, 12 pages, 2017.

[4] Y. Zhou, Y. Bai, J. Li, B. Mao, and T. Li, "Integrated optimization on train control and timetable to minimize net energy consumption of metro lines," Journal of Advanced Transportation, vol. 2018, Article ID 7905820, 19 pages, 2018.

[5] K. Zhao, P. Li, C. Zhang, J. He, X. Li, and J. Liu, "Optimal utilization of adhesion force for heavy-haul electric locomotive based on extremum seeking with sliding mode and asymmetric barrier lyapunov function," Journal of Advanced Transportation, vol. 2019, Article ID 6270515, 15 pages, 2019.

[6] S. Yu and X. Long, "Finite-time consensus for second-order multi-agent systems with disturbances by integral sliding mode," Automatica, vol. 54, no. 54, pp. 158-165, 2015.

[7] C. Zhang, Z. Lin, J. Liu, J. He, H. Wu, and P. Li, “Consensusbased total-amount cooperative tracking control for multimotor locomotive traction system," Journal of the Franklin Institute, vol. 356, no. 2, pp. 819-834, 2019.

[8] C. Zhang, H. Wu, J. He, and C. Xu, "Consensus tracking for multi-motor system via observer based variable structure approach, Journal of the Franklin Institute, vol. 352, no. 8, pp. 3366-3377, 2015.

[9] Z. Zuo and L. Tie, "Distributed robust finite-time nonlinear consensus protocols for multi-agent systems," International Journal of Systems Science, vol. 47, no. 6, pp. 1366-1375, 2016.

[10] J. Khazaei and D. H. Nguyen, "Multi-agent consensus design for heterogeneous energy storage devices with droop control in smart grids," IEEE Transactions on Smart Grid, vol. 10, no. 2, pp. 1395-1404, 2017. 
[11] F. Sun and K. Turkoglu, "Distributed real-time non-linear receding horizon control methodology for multi-agent consensus problems," Aerospace Science \& Technology, vol. 63, pp. 82-90, 2017.

[12] B. Zhang, D. Chang, Z. Li, and D. Ma, "On convergence rate for multi-agent consensus: A community detection algorithm," in 32nd Youth Academic Annual Conference of Chinese Association of Automation (YAC), pp. 72-77, 2017.

[13] W. Zhu, H. Pu, D. Wang, and H. Li, "Event-based consensus of second-order multi-agent systems with discrete time," Automatica, vol. 79, no. 79, pp. 78-83, 2017.

[14] Y. Wu and X. He, "Secure consensus control for multi-agent systems with attacks and communication delays," IEEE/CAA Journal of Automatica Sinica, vol. 4, no. 1, pp. 136-142, 2017.

[15] L. Ding, W. X. Zheng, and G. Guo, "Network-based practical set consensus of multi-agent systems subject to input saturation," Automatica, vol. 89, no. 3, pp. 316-324, 2018.

[16] J. Bai, G. Wen, A. Rahmani, X. Chu, and Y. Yu, "Consensus with a reference state for fractional-order multi-agent systems," International Journal of Systems Science, vol. 47, no. 1, pp. 222$234,2016$.

[17] Z. J. Yang, "Robust consensus tracking control of higher-order uncertain non-linear systems via cascaded finite-time estimation of reference states," IET Control Theory \& Applications, vol. 10, no. 11, pp. 1231-1239, 2016.

[18] H. Wu and H. Su, "Discrete-time positive edge-consensus for undirected and directed nodal networks," IEEE Transactions on Circuits and Systems II: Express Briefs, vol. 65, no. 2, pp. 221-225, 2018.

[19] C. Zhang, Z. Lin, S. X. Yang, and J. He, "Total-amount synchronous control based on terminal sliding-mode control," IEEE Access, vol. 5, pp. 5436-5444, 2017.

[20] S. Lin, Y. Cai, B. Yang, and W. Zhang, "Electrical line-shafting control for motor speed synchronisation using sliding mode controller and disturbance observer," IET Control Theory \& Applications, vol. 11, no. 2, pp. 205-212, 2017.

[21] C. Zhang, Y. Xiao, J. He, and M. Yan, "Improvement of electronic line-shafting control in multi-axis systems," International Journal of Automation and Computing, vol. 15, no. 4, pp. 474481,2018

[22] J. Andert, S. Klein, R. Savelsberg, S. Pischinger, and K. Hameyer, "Virtual shaft: Synchronized motion control for real time testing of automotive powertrains," Control Engineering Practice, vol. 56, pp. 101-110, 2016.

[23] C. Zhang, J. He, L. Jia, and C. Xu, "Virtual line-shafting control for permanent magnet synchronous motor systems using sliding-mode observer," IET Control Theory \& Applications, vol. 9, no. 3, pp. 456-464, 2015.

[24] F. Beltran-Carbajal, A. Valderrabano-Gonzalez, J. C. RosasCaro, and A. Favela-Contreras, "An asymptotic differentiation approach of signals in velocity tracking control of DC motors," Electric Power Systems Research, vol. 122, pp. 218-223, 2015.

[25] H. Wu, C. Zhang, J. He, and K. Zhao, "Distributed fault-tolerant consensus tracking for networked non-identical motors," Journal of Industrial and Management Optimization, vol. 13, no. 2, 53 pages, 2017.

[26] A. Marouf, M. Djemai, C. Sentouh, and P. Pudlo, "A new control strategy of an electric-power-assisted steering system," IEEE Transactions on Vehicular Technology, vol. 61, no. 8, pp. 35743589, 2012.
[27] T. Kamel, D. Abdelkader, B. Said, S. Padmanaban, and A. Iqbal, "Extended kalman filter based sliding mode control of parallelconnected two five-phase PMSM drive system," Electronics, vol. 7, no. 2, pp. 1-19, 2018.

[28] B. Liu, D. J. Hill, Z. Sun, and J. Huang, "Event-triggered control via impulses for exponential stabilization of discrete-time delayed systems and networks," International Journal of Robust and Nonlinear Control, vol. 29, no. 6, pp. 1613-1638, 2019.

[29] B. Liu and D. J. Hill, "Stability via hybrid-event-time Lyapunov function and impulsive stabilization for discrete-time delayed switched systems," SIAM Journal on Control and Optimization, vol. 52, no. 2, pp. 1338-1365, 2014.

[30] Z. Chen, J. He, J. Liu, and Y. Xiong, "Switching delay in selfpowered nonlinear piezoelectric vibration energy harvesting circuit: mechanisms, effects, and solutions," IEEE Transactions on Power Electronics, vol. 34, no. 3, pp. 2427-2440, 2019. 